\title{
Postoperative follow-up programs improve survival in curatively resected gastric and junctional cancer patients: a propensity score matched analysis
}

\author{
Leila Sisic $^{1}$ - Moritz J. Strowitzki ${ }^{1} \cdot$ Susanne Blank $^{1} \cdot$ Henrik Nienhueser $^{1}$ • \\ Sara Dorr ${ }^{1}$ - Georg Martin Haag ${ }^{2}$ Dirk Jäger ${ }^{2}$ Katja Ott $^{3} \cdot$ Markus W. Büchler ${ }^{1}$. \\ Alexis Ulrich ${ }^{1} \cdot$ Thomas Schmidt $^{1}$ (i)
}

Received: 29 May 2017 / Accepted: 15 July 2017 / Published online: 24 July 2017

(C) The International Gastric Cancer Association and The Japanese Gastric Cancer Association 2017

\begin{abstract}
Background To date there is no evidence that more intensive follow-up after surgery for esophagogastric adenocarcinoma translates into improved survival. This study aimed to evaluate the impact of standardized surveillance by a specialized center after resection on survival.

Methods Data of 587 patients were analyzed who underwent curative surgery for esophagogastric adenocarcinoma in our institution. Based on their postoperative surveillance, patients were assigned to either standardized followup (SFU) by the National Center for Tumor Diseases (SFU group) or individual follow-up by other physicians (non-SFU group). Propensity score matching (PSM) was performed to compensate for heterogeneity between groups. Groups were compared regarding clinicopathological findings, recurrence, and impact on survival before and after PSM.

Results Of 587 patients, $32.7 \%$ were in the SFU and $67.3 \%$ in the non-SFU group. Recurrence occurred in $39.4 \%$ of patients and $92.6 \%$ within the first 3 years; $73.6 \%$ were treated, and of those $17.1 \%$ underwent resection. In recurrent patients overall and post-recurrence survival (OS/
\end{abstract}

Electronic supplementary material The online version of this article (doi:10.1007/s10120-017-0751-4) contains supplementary material, which is available to authorized users.

\section{Thomas Schmidt \\ thomas.schmidt1@med.uni-heidelberg.de \\ 1 Department of Surgery, University Hospital Heidelberg, Im Neuenheimer Feld 110, 69120 Heidelberg, Germany \\ 2 Department of Medical Oncology, National Center for Tumor Diseases (NCT), University Hospital Heidelberg,} 69120 Heidelberg, Germany

3 Department of Surgery, RoMed Hospital Rosenheim, 83022 Rosenheim, Germany
PRS) was influenced by diagnostic tools $(p<0.05)$, treatment $(p \leq 0.001)$, and resection of recurrence $(p \leq 0.001)$. Standardized follow-up significantly improved OS (84.9 vs. 38.4 months, $p=0.040$ ) in matched analysis and was an independent positive predictor of OS before and after PSM ( $p=0.034 / 0.013$, respectively).

Conclusion After PSM, standardized follow-up by a specialized center significantly improved OS. Cross-sectional imaging and treatment of recurrence were associated with better outcome. Regular follow-up by cross-sectional imaging especially during the first 3 years should be recommended by national guidelines, since early detection might help select patients for treatment of recurrence and even resection in few designated cases.

Keywords Gastric cancer - Esophageal cancer · Follow-up $\cdot$ Recurrence $\cdot$ Prognosis

$\begin{array}{ll}\text { Abbreviations } \\ \text { SFU } & \text { Standardized follow-up } \\ \text { PSM } & \text { Propensity score matching } \\ \text { OS } & \text { Overall survival } \\ \text { PRS } & \text { Post-recurrence survival } \\ \text { PET } & \text { Positron emission tomography } \\ \text { CT } & \text { Computerized tomography } \\ \text { AEG } & \text { Adenocarcinoma of the esophagogastric junction } \\ \text { UICC } & \text { Union Internationale Contre le Cancer } \\ \text { NCT } & \text { National Center for Tumor Diseases Heidelberg } \\ \text { THG } & \text { Transhiatal extended gastrectomy } \\ \text { MRI } & \text { Magnetic resonance imaging } \\ \text { CTx } & \text { Chemotherapy } \\ \text { CRTx } & \text { Chemoradiotherapy } \\ \text { RTx } & \text { Radiotherapy } \\ \text { HR } & \text { Hazard ratio } \\ \text { CI } & \text { Confidence interval }\end{array}$




\section{Introduction}

It is generally accepted that the recurrence rate after resection of esophagogastric adenocarcinoma ranges from 1.9 to $50.5 \%$ in the literature depending on the tumor stage [1-6], histopathological subtype [7], and type of and response to perioperative treatment [8-11]. Despite the relatively broad worldwide agreement on preoperative staging, perioperative multimodal treatment concepts, and surgical strategies, little consensus has been reached regarding postoperative follow-up [12-15]. Often no national guidelines concerning postoperative patient surveillance exist, and if available, no clear specifications are given on the interval, duration, and mode of follow-up. Surveillance strategies vary from only symptom-based examinations [14-16] to detailed followup plans $[17,18]$ even including regular PET-CT scans in eastern countries [19-21]. European, British, and German guidelines, for example, do not recommend standardized and structured follow-up after gastric cancer resection at all [14-16].

However, a recently published report of the European Registration of Cancer Care (EURECCA) on clinical pathways for esophagogastric adenocarcinoma revealed that in clinical reality many European centers follow more sophisticated surveillance plans including regular cross-sectional imaging and examination of tumor markers [22], even though practice guidelines advocate otherwise. Regular follow-up seems justified, as it not only serves the purpose of detecting recurrent disease, but also offers other important advantages: detection and management of postoperative complications and nutritional issues, psychological support for patients, and data collection for research [23]. The need for more than symptom-driven surveillance is also reflected by the Charter Scaligero on follow-up after gastrectomy for cancer, which was developed by a panel of experts during the 10th International Gastric Cancer Congress in Verona. The Charter states that follow-up should be adapted to the stage of disease, should consist of clinical review and cross-sectional imaging with or without upper gastrointestinal endoscopy, and should be discontinued after 5 years [24].

Many have questioned the oncological benefit of regular surveillance after resection of esophagogastric adenocarcinoma because therapeutic options for recurrent disease are limited, and treatment for recurrence generally consists of palliative chemotherapy. Nowadays probably more attention should be paid to the early diagnosis of mostly asymptomatic recurrence, since-given an adequate performance status-both oncological and surgical treatment has become more aggressive over the last decade even in the palliative setting, including, e.g., triplet combination chemotherapy with more effective but also potentially more toxic agents [25-27], antibodies such as trastuzumab [28] and ramucirumab [29], resection of metastatic disease [30-35], and resection of peritoneal spread with intraperitoneal chemotherapy [36-39].

To date, there is no evidence that standardized follow-up after curative treatment of esophagogastric adenocarcinoma improves the long-term prognosis [23, 40-42]. However, so far not one randomized controlled study evaluating intensive follow-up is available in the literature. Data addressing this problem are limited, retrospective, and of varying quality. Studies comparing intensive vs. less intensive or symptom-driven surveillance could not detect a difference in overall survival, but as there was significant heterogeneity between groups with respect to treatment modalities, age, and tumor stage [43-45], no valid conclusion can be drawn from these results.

Besides the key question whether an intensive follow-up is generally beneficial, at least for a subgroup of patients, it is even more controversial which diagnostic means should be employed during postoperative surveillance and in which intervals [23, 40, 41, 46-49]. Most studies report the highest probability of recurrence during the first 2 years after surgery, whereas relapse after more than 5 years in gastric cancer is reported to be extremely rare $[3,24,42$, 50, 51].

The aim of this retrospective exploratory study was the analysis of two different follow-up programs with respect to their influence on overall survival and survival after recurrence. Second, the effect of the type of diagnostics applied and the type of treatment of recurrence on outcome was analyzed.

\section{Patients and methods}

\section{Study design and patient population}

A total of 972 patients with adenocarcinoma of the stomach or esophago-gastric junction (AEG type I, II, or III according to the Siewert classification) underwent surgery at the University of Heidelberg, Department of Surgery, between 2001 and 2014. Three hundred eighty-five patients were excluded from the analysis because of palliative surgery $(n=68)$, presence of distant metastases (M1, $n=174)$, incomplete resection (Rx/R1, $n=93)$, and postoperative death (in-hospital and/or 90-day mortality, $n=50$ ). Clinicopathological and follow-up data of the remaining 587 patients were collected in a prospective database and analyzed retrospectively. A flow chart of the patient selection process is presented in supplementary Fig. 1. 


\section{Pretreatment staging}

Staging consisted of upper endoscopy with biopsies and a CT scan of the chest and upper abdomen for all patients. Examinations were performed either on an outpatient basis or in the radiological department and endoscopy unit of our institution. Clinical tumor stage and tumor localization were evaluated according to the 7th edition of the UICC staging system. Patients diagnosed before 2010 were reclassified according to the 7th edition of the UICC staging system. The cN category only differentiated between nodalnegative (cN0) and nodal-positive ( $\mathrm{cN}+$ ) disease according to lymph node diameter, shape, and contrast enhancement.

\section{Neoadjuvant treatment}

Two hundred fifty-one patients $(42.8 \%)$ received neoadjuvant treatment. Neoadjuvant treatment consisted of chemotherapy in the majority of cases (95.2\%). Details on neoadjuvant treatment regimens are listed in supplementary Table 1. Patients received treatment on an outpatient basis by either the National Center for Tumor Diseases Heidelberg (NCT) or other treating oncologists.

\section{Surgery}

The surgical approach to tumor resection was chosen depending on tumor localization. Most patients with AEG I underwent a right abdominothoracic en-bloc esophagectomy with a two-field lymphadenectomy (Ivor-Lewis procedure). Patients with AEG III or proximal gastric cancer received a transhiatal extended gastrectomy (THG) with extended D2 lymphadenectomy including left retroperitoneal lymphadenectomy. AEG II patients were treated like either an AEG I or III tumor depending on tumor extension into the esophagus. For tumors in the middle or distal third of the stomach, the standard procedure was a total gastrectomy with D2 lymphadenectomy. In distal tumors, a subtotal gastrectomy was preferred because of quality of life if an adequate proximal resection margin was given. Other surgical procedures included: esophago-gastrectomy $(n=10)$, Merendino procedure $(n=6)$, transmediastinal esophagectomy $(n=6)$, and pancreaticoduodenectomy with total gastrectomy $(n=5)$.

\section{Histopathology and postoperative staging}

Resection specimens were histologically examined in the Department of Pathology. Histopathologic work-up included extent of the primary tumor, regional lymph node spread, presence of distant metastases [(y)pTNM categories according to the UICC classification, 7th edition, 2010], $\mathrm{R}$ category, tumor differentiation (grading), and growth pattern according to Laurén.

\section{Adjuvant treatment}

Adjuvant treatment was administered in the form of perioperative treatment if patients had undergone neoadjuvant treatment. A postoperative adjuvant treatment without previous neoadjuvant therapy was only recommended as an individual treatment decision. Treatment was administered on an outpatient basis by either the NCT or other treating oncologists.

\section{Follow-up}

Patients were followed on an outpatient basis in the NCT according to a standardized protocol every 3 months during the first 2 years, every 6 months during the 3rd and 4 th year, and after 12 months in the 5th year. Structured follow-up was completed 5 years after surgery, and further examinations were based on clinical symptoms. Follow-up examinations included a CT scan every other visit alternating with abdominal sonography and endoscopy every 12-18 months. In the non-SFU group patients underwent individual follow-up by other physicians. At discharge no specification for postoperative follow-up was given, and symptom-driven examinations were recommended according to the German S3 guidelines. Physicians organized follow-up according to their personal discretion. The choice between follow-up at our institution or by other physicians was at the discretion of the patients. The choice of followup data of these patients was obtained by contacting their treating physician and/or the patients themselves. Complete follow-up information was available for all patients. The length of follow-up was calculated from the date of first diagnosis till the last review of patients in February 2017 or time of death. The median follow-up time for surviving patients $(n=337)$ was 64.5 months.

\section{Propensity score matching}

To reduce the effects of selection bias and confounding factors in survival comparison, propensity score matching (PSM) was performed to create comparable groups. Propensity scores were estimated using a multivariable logistic regression model with the follow-up groups as the dependent variables and potential confounders [age, tumor localization, (y)pT category, (y)pN category, neoadjuvant treatment, adjuvant treatment] as covariates. One-toone matching without replacement was performed using a 0.0 caliper width [52]. This allowed us to generate 121 score-matched pairs with either standardized or individual 
follow-up, which were used in subsequent analyses as indicated.

\section{Statistical analysis}

Overall survival (OS) was calculated from time of diagnosis till death or last follow-up date. Post-recurrence survival (PRS) was defined as the interval from diagnosis of recurrence till death or last follow-up. Survival rates were estimated using the Kaplan-Meier method. Differences in survival among groups were calculated using the log-rank test. Prognostic factors were evaluated in a univariate and multivariate analysis using the Cox regression model. To compare categorical variables, we used the $\chi^{2}$ test. The MannWhitney $U$ test was used to compare continuous variables. All tests were two-sided, and a $p$ value $<0.05$ was considered as statistically significant. Analyses were performed using SPSS version 20.0 (SPSS Inc., Chicago, IL, USA).

\section{Results}

\section{Patient demographics, tumor characteristics, and treatment}

The median age at time of diagnosis was 63 (range 27-90) years; 313 patients (53.3\%) were diagnosed with esophageal or junctional and $274(46.7 \%)$ with gastric adenocarcinoma. Detailed information on patient demographics, tumor characteristics, and treatment is summarized in Table 1.

One hundred ninety-two patients $(32.7 \%)$ underwent standardized follow-up (SFU group) and 395 patients (67.3\%) individual follow-up (non-SFU group). Baseline characteristics showed significant heterogeneity between groups: the SFU group was associated with fewer patients aged $\geq 70$ years, more advanced cT, cN, and (y)pT categories, and more frequent administration of neoadjuvant and adjuvant treatment.

To create comparable groups propensity score matching (PSM) was performed. Analysis of heterogeneity after PSM between 121 patients with standardized and 121 patients with individual follow-up revealed almost equal distribution of age, tumor localization, diagnosis, (y)pT and (y) $\mathrm{pN}$ category, and neoadjuvant and adjuvant treatment. Furthermore, no statistically significant difference between the SFU and non-SFU group could be observed with respect to the remaining baseline characteristics (Table 1).

\section{Survival}

Upon last follow-up, 250 of 587 patients (42.6\%) had died. Cause of death was mostly tumor-related $(80.0 \% ; n=200)$; other causes were tumor-unrelated $(9.6 \% ; n=24)$, secondary malignancies $(2.4 \% ; n=6)$, or unknown $(8.0 \%$, $n=20$ ). Median OS from time of diagnosis for all patients was 93.8 (95\% CI 71.4-116.3) months. The estimated 3and 5-year OS rate was 66.2 and $55.7 \%$.

\section{Influence of intensified follow-up on survival}

The median follow-up time for surviving patients was 60.5 months in the SFU $(n=113)$ and 68.5 months in the non-SFU group $(n=224)(p=0.871)$.

No significant difference regarding overall survival comparing all patients $(n=587)$ with standardized (SFU, $n=192$ ) and individual (non-SFU, $n=395$ ) follow-up (median OS: SFU not reached vs. non-SFU 89.9 months; $p=0.490$; Fig. 1a) could be observed.

Subgroup analyses showed a beneficial effect of standardized follow-up on OS for patients with locally advanced tumors [(y)pT3/4, median OS 45.6 vs. 35.2 months, $p=0.096$; supplementary Fig. 2a] and lymph node metastases [(y)pN1/2/3, median OS 44.2 vs. 34.0 months, $p=0.097$; supplementary Fig. 2b], but failed to reach statistical significance.

However, survival analysis of matched patients $(n=242)$ revealed significantly improved overall survival for patients with standardized follow-up (median OS: SFU 84.9 vs. non-SFU 38.4 months, $p=0.040$; Fig. 1b).

There was no significant difference with respect to postrecurrence survival between the SFU and non-SFU group before and after PSM (median PRS: SFU 10.0/9.1 vs. nonSFU 6.0/5.8 months; $p=0.147 / 0.325$, respectively).

\section{Univariate and multivariate analysis for overall survival}

Besides standardized follow-up, which improved the prognosis in the matched patient collective only, age under 70 years as well as lower cT, cN, (y)pT, and (y)pN categories was associated with improved OS in univariate analysis of all and matched patients. Additionally, tumor localization at the stomach and resection by subtotal gastrectomy were significant positive prognostic factors before PSM (Table 2).

Multivariate Cox regression analysis included the following factors: follow-up, age, gender, diagnosis, grading, Laurén classification, cT category, cN category, neoadjuvant treatment, surgery, (y)pT category, (y)pN category, and adjuvant treatment.

Independent positive prognosticators for OS of all and matched patients were: age under 70 years, lower (y)pN category, and standardized follow-up. cT category was an independent prognostic factor before PSM, whereas $\mathrm{cN}$ 
Table 1 Baseline characteristics of patients with individual (non-SFU) and standardized follow-up (SFU) before and after propensity score matching

\begin{tabular}{|c|c|c|c|c|c|c|c|c|c|c|}
\hline & \multicolumn{5}{|c|}{ All patients $(n=587)$} & \multicolumn{5}{|c|}{ Matched patients $(n=242)$} \\
\hline & \multicolumn{2}{|c|}{$\begin{array}{l}\text { Non-SFU } \\
(n=395)\end{array}$} & \multicolumn{2}{|c|}{$\begin{array}{l}\text { SFU } \\
(n=192)\end{array}$} & \multirow[t]{2}{*}{$p$} & \multicolumn{2}{|c|}{$\begin{array}{l}\text { Non-SFU } \\
(n=121)\end{array}$} & \multicolumn{2}{|c|}{$\begin{array}{l}\text { SFU } \\
(n=121)\end{array}$} & \multirow[t]{2}{*}{$p$} \\
\hline & $n$ & $\%$ & $n$ & $\%$ & & $n$ & $\%$ & $n$ & $\%$ & \\
\hline Age at diagnosis (years) & & & & & 0.001 & & & & & 1.000 \\
\hline$\leq 45$ & 32 & 8.1 & 11 & 5.7 & & 3 & 2.5 & 3 & 2.5 & \\
\hline $46-69$ & 223 & 56.5 & 140 & 72.9 & & 88 & 72.7 & 88 & 72.7 & \\
\hline$\geq 70$ & 140 & 35.4 & 41 & 21.4 & & 30 & 24.8 & 30 & 24.8 & \\
\hline Gender & & & & & 0.947 & & & & & 0.228 \\
\hline Male & 287 & 72.7 & 139 & 72.4 & & 96 & 79.3 & 88 & 72.7 & \\
\hline Female & 108 & 27.3 & 53 & 27.6 & & 25 & 20.7 & 33 & 27.3 & \\
\hline Localization & & & & & 0.523 & & & & & 1.000 \\
\hline Esophagus/EGJ & 207 & 52.4 & 106 & 55.2 & & 71 & 58.7 & 71 & 58.7 & \\
\hline Stomach & 188 & 47.6 & 86 & 44.8 & & 50 & 41.3 & 50 & 41.3 & \\
\hline Diagnosis & & & & & 0.883 & & & & & 0.987 \\
\hline AEG I & 84 & 21.3 & 44 & 22.9 & & 31 & 25.6 & 30 & 24.8 & \\
\hline AEG II & 97 & 24.6 & 47 & 24.5 & & 31 & 25.6 & 33 & 27.3 & \\
\hline AEG III & 26 & 6.6 & 15 & 7.8 & & 9 & 7.4 & 8 & 6.6 & \\
\hline Gastric cancer & 188 & 47.6 & 86 & 44.8 & & 50 & 41.3 & 50 & 41.3 & \\
\hline Grading & & & & & 0.101 & & & & & 0.646 \\
\hline Low grade $(\mathrm{G} 1 / 2)$ & 146 & 37.0 & 57 & 29.7 & & 44 & 36.4 & 41 & 33.9 & \\
\hline High grade (G3/4) & 234 & 59.2 & 125 & 65.1 & & 71 & 58.7 & 75 & 62.0 & \\
\hline Missing data & 15 & 3.8 & 10 & 5.2 & & 6 & 5.0 & 5 & 4.1 & \\
\hline Laurén classification & & & & & 0.108 & & & & & 0.171 \\
\hline Intestinal & 211 & 53.4 & 91 & 47.4 & & 73 & 60.3 & 63 & 52.1 & \\
\hline Non-intestinal & 152 & 38.5 & 88 & 45.8 & & 39 & 32.2 & 49 & 40.5 & \\
\hline Missing data & 32 & 8.1 & 13 & 6.8 & & 9 & 7.4 & 9 & 7.4 & \\
\hline cT category & & & & & $<0.001$ & & & & & 0.418 \\
\hline $\mathrm{cT} 1$ & 49 & 12.4 & 8 & 4.2 & & 4 & 3.3 & 7 & 5.8 & \\
\hline $\mathrm{cT} 2$ & 120 & 30.4 & 36 & 18.8 & & 27 & 22.3 & 30 & 24.8 & \\
\hline cT3 & 185 & 46.8 & 124 & 64.6 & & 77 & 63.6 & 68 & 56.2 & \\
\hline cT4 & 18 & 4.6 & 18 & 9.4 & & 7 & 5.8 & 12 & 9.9 & \\
\hline Missing data & 23 & 5.8 & 6 & 3.1 & & 6 & 5.0 & 4 & 3.3 & \\
\hline cN category & & & & & $<0.001$ & & & & & 0.585 \\
\hline $\mathrm{cN} 0$ & 214 & 54.2 & 66 & 34.4 & & 48 & 39.7 & 53 & 43.8 & \\
\hline $\mathrm{cN}+$ & 159 & 40.3 & 121 & 63.0 & & 68 & 56.2 & 65 & 53.7 & \\
\hline Missing data & 22 & 5.6 & 5 & 2.6 & & 5 & 4.1 & 3 & 2.5 & \\
\hline Neoadjuvant treatment & & & & & $<0.001$ & & & & & 1.000 \\
\hline Primary surgery & 268 & 67.8 & 68 & 35.4 & & 54 & 44.6 & 54 & 44.6 & \\
\hline Neoadjuvant treatment & 127 & 32.2 & 124 & 64.6 & & 67 & 55.4 & 67 & 55.4 & \\
\hline \multicolumn{3}{|c|}{ Type of neoadjuvant treatment } & & & 0.522 & & & & & 0.247 \\
\hline Chemotherapy & 119 & 93.7 & 120 & 96.8 & & 61 & 91.0 & 65 & 97.0 & \\
\hline Chemoradiotherapy & 6 & 4.7 & 3 & 2.4 & & 4 & 6.0 & 2 & 3.0 & \\
\hline Radiotherapy & 2 & 1.6 & 1 & 0.8 & & 2 & 3.0 & 0 & 0.0 & \\
\hline Surgery & & & & & 0.510 & & & & & 0.352 \\
\hline Subtotal gastrectomy & 106 & 26.8 & 40 & 20.8 & & 28 & 23.1 & 25 & 20.7 & \\
\hline Total gastrectomy & 92 & 23.3 & 43 & 22.4 & & 26 & 21.5 & 21 & 17.4 & \\
\hline THG & 88 & 22.3 & 47 & 24.5 & & 25 & 20.7 & 33 & 27.3 & \\
\hline Ivor Lewis procedure & 91 & 23.0 & 53 & 27.6 & & 34 & 28.1 & 39 & 32.2 & \\
\hline Other & 18 & 4.6 & 9 & 4.7 & & 8 & 6.6 & 3 & 2.5 & \\
\hline (y)pT category & & & & & 0.019 & & & & & 0.995 \\
\hline
\end{tabular}


Table 1 (continued)

\begin{tabular}{|c|c|c|c|c|c|c|c|c|c|c|}
\hline & \multicolumn{5}{|c|}{ All patients $(n=587)$} & \multicolumn{5}{|c|}{ Matched patients $(n=242)$} \\
\hline & \multicolumn{2}{|c|}{$\begin{array}{l}\text { Non-SFU } \\
(n=395)\end{array}$} & \multicolumn{2}{|c|}{$\begin{array}{l}\text { SFU } \\
(n=192)\end{array}$} & \multirow[t]{2}{*}{$p$} & \multicolumn{2}{|c|}{$\begin{array}{l}\text { Non-SFU } \\
(n=121)\end{array}$} & \multicolumn{2}{|c|}{$\begin{array}{l}\text { SFU } \\
(n=121)\end{array}$} & \multirow[t]{2}{*}{$p$} \\
\hline & $n$ & $\%$ & $n$ & $\%$ & & $n$ & $\%$ & $n$ & $\%$ & \\
\hline (y)pT0 & 18 & 4.6 & 11 & 5.7 & & 6 & 5.0 & 6 & 5.0 & \\
\hline (y)pT1 & 101 & 25.6 & 28 & 14.6 & & 20 & 16.5 & 20 & 16.5 & \\
\hline (y)pT2 & 70 & 17.7 & 29 & 15.1 & & 18 & 14.9 & 18 & 14.9 & \\
\hline (y)pT3 & 183 & 46.3 & 108 & 56.3 & & 66 & 54.5 & 64 & 52.9 & \\
\hline (y)pT4 & 23 & 5.8 & 16 & 8.3 & & 11 & 9.1 & 13 & 10.7 & \\
\hline (y)pN category & & & & & 0.138 & & & & & 1.000 \\
\hline (y)pN0 & 198 & 50.1 & 78 & 40.6 & & 58 & 47.9 & 58 & 47.9 & \\
\hline (y)pN1 & 63 & 15.9 & 37 & 19.3 & & 20 & 16.5 & 20 & 16.5 & \\
\hline (y)pN2 & 56 & 14.2 & 37 & 19.3 & & 13 & 10.7 & 13 & 10.7 & \\
\hline (y)pN3 & 78 & 19.7 & 40 & 20.8 & & 30 & 24.8 & 30 & 24.8 & \\
\hline Adjuvant treatment & & & & & $<0.001$ & & & & & 1.000 \\
\hline Not administered & 286 & 72.4 & 94 & 49.0 & & 67 & 55.4 & 67 & 55.4 & \\
\hline Administered & 109 & 27.6 & 98 & 51.0 & & 54 & 44.6 & 54 & 44.6 & \\
\hline Type of adjuvant treatment & & & & & 0.046 & & & & & 0.235 \\
\hline Chemotherapy & 93 & 85.3 & 92 & 93.9 & & 49 & 90.7 & 51 & 94.4 & \\
\hline Chemoradiotherapy & 16 & 14.7 & 6 & 6.1 & & 5 & 9.3 & 3 & 5.6 & \\
\hline
\end{tabular}

Bold values indicate a significance-level of $p<0.05$

$S F U$ standardized follow-up, EGJ esophagogastric junction, $A E G$ adenocarcinoma of the esophagogastric junction, $T H G$ transhiatal extended gastrectomy
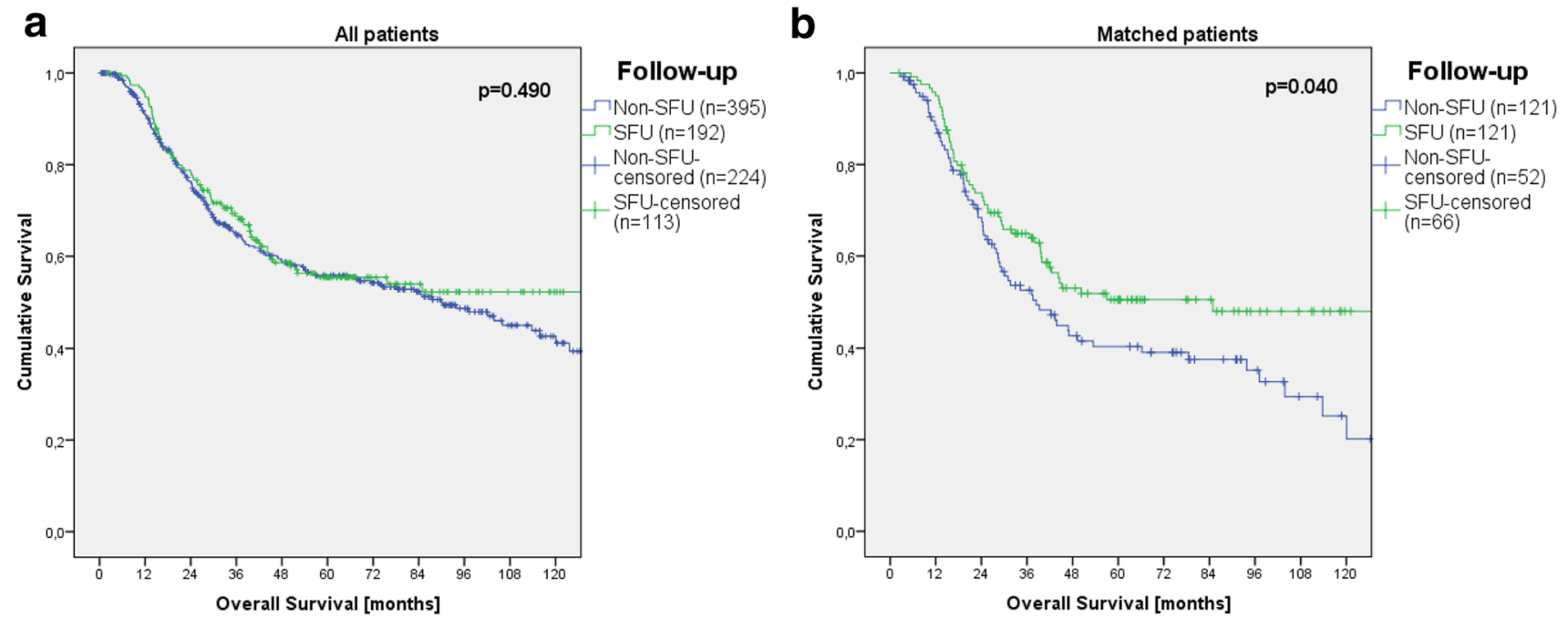

Fig. 1 Influence of standardized follow-up (SFU) on overall survival a before and $\mathbf{b}$ after propensity score matching (PSM)

category was an independent prognostic factor after PSM (Table 3).

\section{Recurrence}

Of 587 patients, 231 (39.4\%) developed recurrence; $82.2 \%$ of all recurrences $(n=190)$ were diagnosed within the first 24 months and $92.6 \%$ within 36 months after surgery; $43.3 \%$ of patients $(n=100)$ were asymptomatic when recurrence was detected. Recurrence rate and risk for recurrence were increased in case of proximal tumor localization, advanced (y)pT category, and advanced (y) pN category (all $p<0.001$ ) (supplementary Table 2). Diagnosis of recurrence was made by cross-sectional imaging (CT or MRI) in the majority of cases (82.3\%), and histological confirmation was possible in $43.3 \%(n=100)$. Most patients $(82.7 \%)$ relapsed in the form of distant recurrence, while less than one third $(28.1 \%)$ showed locoregional 
Table 2 Univariate Cox regression analysis for overall survival before and after propensity score matching

\begin{tabular}{|c|c|c|c|c|c|c|c|c|}
\hline & \multicolumn{4}{|c|}{ All patients $(n=587)$} & \multicolumn{4}{|c|}{ Matched patients $(n=242)$} \\
\hline & $n$ & HR & $95 \% \mathrm{CI}$ & $p$ & $n$ & HR & $95 \% \mathrm{CI}$ & $p$ \\
\hline Follow-up & & & & 0.490 & & & & 0.040 \\
\hline Non-SFU & 395 & 1 & & & 121 & 1 & & \\
\hline SFU & 192 & 0.91 & $0.70-1.19$ & & 121 & 0.69 & $0.49-0.99$ & \\
\hline Age at diagnosis (years) & & & & $<0.001$ & & & & $<0.001$ \\
\hline$\leq 45$ & 43 & 1 & & & 6 & 1 & & \\
\hline $46-69$ & 363 & 0.97 & $0.57-1.66$ & & 176 & 0.90 & $0.28-2.86$ & \\
\hline$\geq 70$ & 181 & 1.66 & $0.96-2.86$ & & 60 & 2.03 & $0.63-6.56$ & \\
\hline Gender & & & & 0.194 & & & & 0.893 \\
\hline Male & 426 & 1 & & & 184 & 1 & & \\
\hline Female & 161 & 0.83 & $0.62-1.10$ & & 58 & 0.97 & $0.64-1.47$ & \\
\hline Localization & & & & $<0.001$ & & & & 0.079 \\
\hline Esophagus/EGJ & 313 & 1 & & & 142 & 1 & & \\
\hline Stomach & 274 & 0.60 & $0.46-0.77$ & & 100 & 0.72 & $0.50-1.04$ & \\
\hline Diagnosis & & & & 0.001 & & & & 0.290 \\
\hline AEG I & 128 & 1 & & & 61 & 1 & & \\
\hline AEG II & 144 & 1.05 & $0.75-1.47$ & & 64 & 0.95 & $0.59-1.51$ & \\
\hline AEG III & 41 & 1.14 & $0.69-1.86$ & & 17 & 1.23 & $0.62-2.43$ & \\
\hline Gastric cancer & 274 & 0.62 & $0.45-0.86$ & & 100 & 0.72 & $0.46-1.13$ & \\
\hline Grading & & & & 0.177 & & & & 0.954 \\
\hline Low grade (G1/2) & 203 & 1 & & & 85 & 1 & & \\
\hline High grade (G3/4) & 359 & 1.20 & $0.92-1.56$ & & 146 & 0.99 & $0.69-1.43$ & \\
\hline Laurén classification & & & & 0.729 & & & & 0.447 \\
\hline Intestinal & 302 & 1 & & & 136 & 1 & & \\
\hline Non-intestinal & 240 & 1.05 & $0.81-1.36$ & & 88 & 0.86 & $0.59-1.26$ & \\
\hline cT category & & & & $<0.001$ & & & & 0.008 \\
\hline cT1 & 57 & 1 & & & 11 & 1 & & \\
\hline $\mathrm{cT} 2$ & 156 & 3.04 & $1.51-6.15$ & & 57 & 3.14 & $0.75-13.27$ & \\
\hline cT3 & 309 & 5.02 & $2.56-9.85$ & & 145 & 5.08 & $1.24-20.77$ & \\
\hline cT4 & 36 & 6.81 & $3.05-15.20$ & & 19 & 6.65 & $1.48-29.96$ & \\
\hline $\mathrm{cN}$ category & & & & $<0.001$ & & & & 0.003 \\
\hline $\mathrm{cNO}$ & 280 & 1 & & & 101 & 1 & & \\
\hline $\mathrm{cN}+$ & 280 & 1.63 & $1.26-2.11$ & & 133 & 1.75 & $1.20-2.54$ & \\
\hline Neoadjuvant treatment & & & & 0.232 & & & & 0.634 \\
\hline Primary surgery & 336 & 1 & & & 108 & 1 & & \\
\hline Neoadjuvant treatment & 251 & 1.17 & $0.91-1.51$ & & 134 & 1.09 & $0.76-1.56$ & \\
\hline Surgery & & & & $<0.001$ & & & & 0.123 \\
\hline Subtotal gastrectomy & 146 & 1 & & & 53 & 1 & & \\
\hline Total gastrectomy & 135 & 1.63 & $1.09-2.45$ & & 47 & 1.46 & $0.81-2.65$ & \\
\hline THG & 135 & 2.26 & $1.55-3.30$ & & 58 & 1.61 & $0.94-2.75$ & \\
\hline Ivor Lewis procedure & 144 & 1.92 & $1.30-2.83$ & & 73 & 1.60 & $0.95-2.71$ & \\
\hline Other & 27 & 2.92 & $1.64-5.19$ & & 11 & 2.92 & $1.24-6.88$ & \\
\hline (y)pT category & & & & $<0.001$ & & & & $<0.001$ \\
\hline (y)pT0 & 29 & 1 & & & 12 & 1 & & \\
\hline (y)pT1 & 129 & 0.55 & $0.26-1.18$ & & 40 & 0.97 & $0.32-2.97$ & \\
\hline (y)pT2 & 99 & 1.23 & $0.60-2.55$ & & 36 & 1.19 & $0.39-3.63$ & \\
\hline (y)pT3 & 291 & 2.01 & $1.03-3.94$ & & 130 & 2.13 & $0.78-5.83$ & \\
\hline (y)pT4 & 39 & 3.95 & $1.84-8.48$ & & 24 & 3.86 & $1.29-11.58$ & \\
\hline (y)pN category & & & & $<0.001$ & & & & $<0.001$ \\
\hline (y)pN0 & 276 & 1 & & & 116 & 1 & & \\
\hline
\end{tabular}


Table 2 (continued)

\begin{tabular}{|c|c|c|c|c|c|c|c|c|}
\hline & \multicolumn{4}{|c|}{ All patients $(n=587)$} & \multicolumn{4}{|c|}{ Matched patients $(n=242)$} \\
\hline & $n$ & HR & $95 \% \mathrm{CI}$ & $p$ & $n$ & HR & $95 \% \mathrm{CI}$ & $p$ \\
\hline (y)pN1 & 100 & 1.63 & $1.10-2.40$ & & 40 & 1.49 & $0.87-2.56$ & \\
\hline (y)pN2 & 93 & 2.87 & $2.02-4.09$ & & 26 & 2.53 & $1.47-4.36$ & \\
\hline (y)pN3 & 118 & 5.05 & $3.66-6.95$ & & 60 & 3.63 & $2.36-5.58$ & \\
\hline Adjuvant treatment & & & & 0.766 & & & & 0.179 \\
\hline Not administered & 380 & 1 & & & 134 & 1 & & \\
\hline Administered & 207 & 0.96 & $0.73-1.26$ & & 108 & 0.78 & $0.54-1.12$ & \\
\hline
\end{tabular}

Bold values indicate a significance-level of $p<0.05$

$H R$ hazard ratio, $C I$ confidence interval, $S F U$ standardized follow-up, $E G J$ esophagogastric junction, $A E G$ adenocarcinoma of the esophagogastric junction, $T H G$ transhiatal extended gastrectomy

Table 3 Multivariate Cox regression analysis for overall survival before and after propensity score matching

\begin{tabular}{|c|c|c|c|c|c|c|c|c|}
\hline & \multicolumn{4}{|c|}{ All patients $(n=587)$} & \multicolumn{4}{|c|}{ Matched patients $(n=242)$} \\
\hline & $n$ & HR & $95 \% \mathrm{CI}$ & $p$ & $n$ & HR & $95 \% \mathrm{CI}$ & $p$ \\
\hline \multicolumn{9}{|l|}{ Age at diagnosis (years) } \\
\hline$\leq 45$ (reference) & 43 & 1 & & 0.001 & 6 & 1 & & $<0.001$ \\
\hline $46-69$ & 363 & 1.32 & $0.75-2.33$ & 0.330 & 176 & 1.43 & $0.44-4.61$ & 0.555 \\
\hline$\geq 70$ & 181 & 2.20 & $1.23-3.93$ & 0.008 & 60 & 3.34 & $1.01-11.12$ & 0.049 \\
\hline \multicolumn{9}{|l|}{ cT category } \\
\hline cT1 (reference) & 57 & 1 & & $<0.001$ & NA & & & \\
\hline $\mathrm{cT} 2$ & 156 & 2.42 & $1.15-5.13$ & 0.021 & & & & \\
\hline cT3 & 309 & 3.78 & $1.81-7.88$ & $<0.001$ & & & & \\
\hline cT4 & 36 & 5.36 & $2.19-13.12$ & $<0.001$ & & & & \\
\hline $\mathrm{cN}$ category & & & & & & & & 0.043 \\
\hline cN0 (reference) & NA & & & & 101 & 1 & & \\
\hline $\mathrm{cN}+$ & & & & & 133 & 1.56 & $1.01-2.39$ & \\
\hline \multicolumn{9}{|l|}{ (y)pN category } \\
\hline (y)pN0 (reference) & 276 & 1 & & $<0.001$ & 116 & 1 & & $<0.001$ \\
\hline (y)pN1 & 100 & 1.41 & $0.93-2.16$ & 0.109 & 40 & 1.33 & $0.75-2.35$ & 0.324 \\
\hline (y)pN2 & 93 & 2.25 & $1.52-3.32$ & $<0.001$ & 26 & 2.12 & $1.13-3.98$ & 0.019 \\
\hline (y)pN3 & 118 & 3.69 & $2.60-5.23$ & $<0.001$ & 60 & 2.95 & $1.82-4.77$ & $<0.001$ \\
\hline \multicolumn{9}{|l|}{ Follow-up } \\
\hline Non-SFU (reference) & 395 & 1 & & & 121 & 1 & & \\
\hline SFU & 192 & 0.72 & $0.53-0.97$ & 0.034 & 121 & 0.61 & $0.42-0.90$ & 0.013 \\
\hline
\end{tabular}

Bold values indicate a significance-level of $p<0.05$

Factors included: follow-up, age, gender, diagnosis, grading, Laurén classification, cT category, cN category, neoadjuvant treatment, surgery, (y)pT category, (y)pN category, adjuvant treatment

$H R$ hazard ratio, $C I$ confidence interval, $S F U$ standardized follow-up, $N A$ not applicable recurrence. The most frequent pattern of distant recurrence was distant lymphatic spread $(36.4 \%)$, followed by peritoneal recurrence $(30.7 \%)$, liver $(25.5 \%)$, lung $(12.1 \%)$, and bone metastases $(10.0 \%)$. Less frequent sites $(<5 \%)$ included the brain, soft tissue, adrenal, and ovarian metastases. In 101 patients $(43.7 \%)$, recurrence was detected at multiple sites (supplementary Fig. 3).

One hundred seventy patients $(73.6 \%)$ were treated for recurrence, while 61 patients did not receive any treatment for recurrence at all $(26.4 \%)$. Treatment of recurrence was mostly with palliative intent $(85.3 \%)$. Twenty-nine of the patients who received treatment $(17.1 \%)$ underwent resection of recurrence with or without additional treatment; 141 patients $(82.9 \%)$ received only chemotherapy (CTx), chemoradiotherapy (CRTx), radiotherapy (RTx), or any combination of these treatment modalities. Details on time of diagnosis, diagnostic means for detection, patterns, and treatment of recurrence are listed in Table 4.

Eighty-three patients $(43.2 \%)$ in the SFU group and $148(37.5 \%)$ in the non-SFU group developed recurrence ( $p=0.180)$. No significant differences between the SFU and non-SFU group could be detected regarding time of diagnosis, symptoms, diagnostic means for detection, and pattern of recurrence, except for liver metastases, which 
Table 4 Time of diagnosis, diagnostic means for detection, pattern, and treatment of recurrence in patients with individual (non-SFU) and standardized follow-up (SFU)

\begin{tabular}{|c|c|c|c|c|c|c|c|}
\hline Interval till recurrence & \multicolumn{2}{|c|}{ Non-SFU $(n=148)$} & \multicolumn{2}{|c|}{$\mathrm{SFU}(n=83)$} & \multicolumn{2}{|c|}{ Total $(n=231)$} & $\frac{p}{0.362}$ \\
\hline$<12$ months & 76 & $51.4 \%$ & 52 & $62.7 \%$ & 128 & $55.4 \%$ & \\
\hline 12-24 months & 42 & $28.4 \%$ & 20 & $24.1 \%$ & 62 & $26.8 \%$ & \\
\hline 24-36 months & 17 & $11.5 \%$ & 7 & $8.4 \%$ & 24 & $10.4 \%$ & \\
\hline$>36$ months & 13 & $8.8 \%$ & 4 & $4.8 \%$ & 17 & $7.4 \%$ & \\
\hline Symptomatic recurrence & & & & & & & 0.276 \\
\hline No & 53 & $35.8 \%$ & 47 & $56.6 \%$ & 100 & $43.3 \%$ & \\
\hline Yes & 53 & $35.8 \%$ & 34 & $41.0 \%$ & 87 & $37.7 \%$ & \\
\hline Missing data & 42 & $28.4 \%$ & 2 & $2.4 \%$ & 44 & $19.0 \%$ & \\
\hline Diagnostic means & & & & & & & 0.336 \\
\hline Clinically/sonography & 13 & $8.8 \%$ & 5 & $6.0 \%$ & 18 & $7.8 \%$ & \\
\hline Endoscopy & 5 & $3.4 \%$ & 2 & $2.4 \%$ & 7 & $3.0 \%$ & \\
\hline Imaging (CT or MRI) & 88 & $59.5 \%$ & 65 & $78.3 \%$ & 153 & $66.2 \%$ & \\
\hline Imaging and endoscopy & 26 & $17.6 \%$ & 11 & $13.3 \%$ & 37 & $16.0 \%$ & \\
\hline Missing data & 16 & $10.8 \%$ & 0 & $0.0 \%$ & 16 & $6.9 \%$ & \\
\hline Cross-sectional imaging & & & & & & & 0.247 \\
\hline No & 18 & $12.2 \%$ & 7 & $8.4 \%$ & 25 & $10.8 \%$ & \\
\hline Yes & 114 & $77.0 \%$ & 76 & $91.6 \%$ & 190 & $82.3 \%$ & \\
\hline Missing data & 16 & $10.8 \%$ & 0 & $0.0 \%$ & 16 & $6.9 \%$ & \\
\hline Histologically confirmed & & & & & & & 0.311 \\
\hline No & 67 & $45.3 \%$ & 48 & $57.8 \%$ & 115 & $49.8 \%$ & \\
\hline Yes & 65 & $43.9 \%$ & 35 & $42.2 \%$ & 100 & $43.3 \%$ & \\
\hline Missing data & 16 & $10.8 \%$ & 0 & $0.0 \%$ & 16 & $6.9 \%$ & \\
\hline \multicolumn{8}{|l|}{ Pattern of recurrence } \\
\hline Locoregional & 45 & $30.4 \%$ & 20 & $24.1 \%$ & 65 & $28.1 \%$ & 0.129 \\
\hline Distant & 117 & $79.1 \%$ & 75 & $90.4 \%$ & 192 & $83.1 \%$ & 0.586 \\
\hline Lymphatic & 50 & $33.8 \%$ & 34 & $41.0 \%$ & 84 & $36.4 \%$ & 0.621 \\
\hline Peritoneal & 45 & $30.4 \%$ & 26 & $31.3 \%$ & 71 & $30.7 \%$ & 0.703 \\
\hline Liver & 46 & $31.1 \%$ & 13 & $15.7 \%$ & 59 & $25.5 \%$ & 0.002 \\
\hline Lung & 13 & $8.8 \%$ & 15 & $18.1 \%$ & 28 & $12.1 \%$ & 0.077 \\
\hline Bone & 15 & $10.1 \%$ & 8 & $9.6 \%$ & 23 & $10.0 \%$ & 0.704 \\
\hline Brain & 8 & $5.4 \%$ & 2 & $2.4 \%$ & 10 & $4.3 \%$ & 0.220 \\
\hline Adrenal & 3 & $2.0 \%$ & 5 & $6.0 \%$ & 8 & $3.5 \%$ & 0.154 \\
\hline Soft tissue & 4 & $2.7 \%$ & 3 & $3.6 \%$ & 7 & $3.0 \%$ & 0.806 \\
\hline Ovarian & 4 & $2.7 \%$ & 3 & $3.6 \%$ & 7 & $3.0 \%$ & 0.806 \\
\hline Other & 2 & $1.4 \%$ & 1 & $1.2 \%$ & 3 & $1.3 \%$ & 0.855 \\
\hline Multiple sites & 69 & $46.6 \%$ & 32 & $38.6 \%$ & 101 & $43.7 \%$ & 0.056 \\
\hline Missing data & 15 & $10.1 \%$ & 0 & $0.0 \%$ & 15 & $6.5 \%$ & \\
\hline Treatment of recurrence & & & & & & & 0.001 \\
\hline No & 50 & $33.8 \%$ & 11 & $13.3 \%$ & 61 & $26.4 \%$ & \\
\hline \multirow[t]{2}{*}{ Yes } & 98 & $66.2 \%$ & 72 & $86.7 \%$ & 170 & $73.6 \%$ & \\
\hline & \multicolumn{2}{|c|}{ Non-SFU $(n=98)$} & \multicolumn{2}{|c|}{$\mathrm{SFU}(n=72)$} & \multicolumn{2}{|c|}{ Total $(n=170)$} & $p$ \\
\hline \multicolumn{8}{|l|}{ Therapy of recurrence } \\
\hline Intention of treatment & & & & & & & 0.135 \\
\hline Palliative & 87 & $88.8 \%$ & 58 & $80.6 \%$ & 145 & $85.3 \%$ & \\
\hline Curative & 11 & $11.2 \%$ & 14 & $19.4 \%$ & 25 & $14.7 \%$ & \\
\hline Resection of recurrence & & & & & & & 0.767 \\
\hline No (CTx/CRTx/RTx only) & 82 & $83.7 \%$ & 59 & $81.9 \%$ & 141 & $82.9 \%$ & \\
\hline
\end{tabular}


Table 4 (continued)

\begin{tabular}{|c|c|c|c|c|c|c|c|}
\hline \multirow[b]{2}{*}{ Yes $( \pm \mathrm{CTx} / \mathrm{CRTx} / \mathrm{RTx})$} & \multicolumn{2}{|c|}{ Non-SFU $(n=98)$} & \multicolumn{2}{|c|}{$\operatorname{SFU}(n=72)$} & \multicolumn{2}{|c|}{ Total $(n=170)$} & \multirow[t]{2}{*}{$p$} \\
\hline & 16 & $16.3 \%$ & 13 & $18.1 \%$ & 29 & $17.1 \%$ & \\
\hline Type of therapy & & & & & & & 0.300 \\
\hline Resection & 6 & $6.1 \%$ & 9 & $12.5 \%$ & 15 & $8.8 \%$ & \\
\hline Resection + CTx/CRTx/RTx & 10 & $10.2 \%$ & 4 & $5.6 \%$ & 14 & $8.2 \%$ & \\
\hline CTx only & 59 & $60.2 \%$ & 46 & $63.9 \%$ & 105 & $61.8 \%$ & \\
\hline CRTx/RTx $( \pm$ CTx $)$ & 23 & $23.5 \%$ & 13 & $0.0 \%$ & 36 & $21.2 \%$ & \\
\hline
\end{tabular}

Bold values indicate a significance-level of $p<0.05$

$S F U$ standardized follow-up, $C T$ computed tomography, MRI magnetic resonance imaging, CTx chemotherapy, $C R T x$ chemoradiotherapy, $R T x$ radiotherapy, + and / or \pm with or without

were more frequent in the non-SFU group (31.1 vs. $15.7 \%$, $p=0.002)$. Patients in the SFU group were more likely to receive treatment for recurrence than patients in the nonSFU group ( 86.7 vs. $66.2 \%$; $p=0.001)$. However, there was no difference with respect to type of treatment (Table 4).

\section{Diagnosis, pattern, and treatment of recurrence and survival}

Of 231 patients with recurrence, 200 had died at last follow-up. Cause of death was tumor-related in all cases $(n=200)$. For patients with recurrence, median overall survival from time of diagnosis was 24.2 (95\% CI 21.3-27.2) months, and median post-recurrence survival was 7.1 (95\% CI 5.2-9.0) months.

OS and PRS were considerably improved by treatment or recurrence (Fig. 2a, b), surgical resection of recurrence (Fig. 2c, d), and especially resection combined with CTx and/or CRTx and/or RTx (Fig. 2e, f). Additionally, improved OS and PRS were linked to asymptomatic recurrence (supplementary Fig. 4a, b), diagnostic means for detection of recurrence (supplementary Fig. 5a, b), and particularly detection of recurrence by cross-sectional imaging (supplementary Fig. 5c, d). Survival analyses for patients with recurrent disease regarding factors associated with the diagnosis, pattern, and treatment of recurrence are summarized in Table 5.

Multivariate analysis for PRS identified symptomatic (HR 1.73, $p=0.005$ ) and peritoneal recurrence (HR 1.54, $p=0.040)$ as negative independent prognosticators, while resection of recurrence significantly improved PRS (HR $0.36, p<0.001$ ) (supplementary Table 3 ).

\section{Discussion}

In the absence of randomized controlled trials, the discussion on the need, schedule, and recommended examinations for follow-up after resection of esophagogastric cancer has been completely controversial worldwide [12-15, 24].
Our study is the first one to compare intensive follow-up by a specialized center with individual follow-up of other physicians after reducing heterogeneity between groups by propensity score matching. We could show that patients are likely to benefit from standardized surveillance by a specialized center after complete resection of esophagogastric adenocarcinoma if baseline characteristics are comparable between groups. The lack of survival benefit from intensive follow-up in our entire patient collective can be explained by more advanced (y)pT and (y)pN categories in the SFU group. Patients with more advanced tumor stages are more likely to be subjected to intensive follow-up than patients with early stage disease $[43,44,46]$. We believe that this selection bias could explain why other series failed to show a beneficial effect of intensive postoperative surveillance on survival [43-45]. Besides, even general practitioners or other outpatient physicians seem to frequently arrange cross-sectional imaging during follow-up for patients with esophagogastric cancer. Apparently most patients in Germany receive relatively intensive surveillance including cross-sectional imaging despite the German guidelines not recommending any regular follow-up examinations in esophagogastric cancer at all [16, 22]. This might be symptomatic of another reason for regular follow-up, namely the patients' wishes. Nowadays, patients are well informed and probably not satisfied with reassessment only in case of clinical symptoms. It seems as if repeated follow-up examinations do not lead to discomfort but rather reassurance of the patients. Furthermore, the literature confirms that early asymptomatic diagnosis of recurrence is associated with improved prognosis [53, 54].

According to our data, cross-sectional imaging seems to be the most effective examination technique for detection of recurrence. In our series, mostly CT scans were performed, and recurrence was rarely discovered by endoscopy. Since-as in our analysis-the most frequent pattern of recurrence after $\mathrm{R} 0$ resection of esophagogastric adenocarcinoma is distant and only a small percentage of patients show local relapse $[4,42,55]$, cross-sectional 

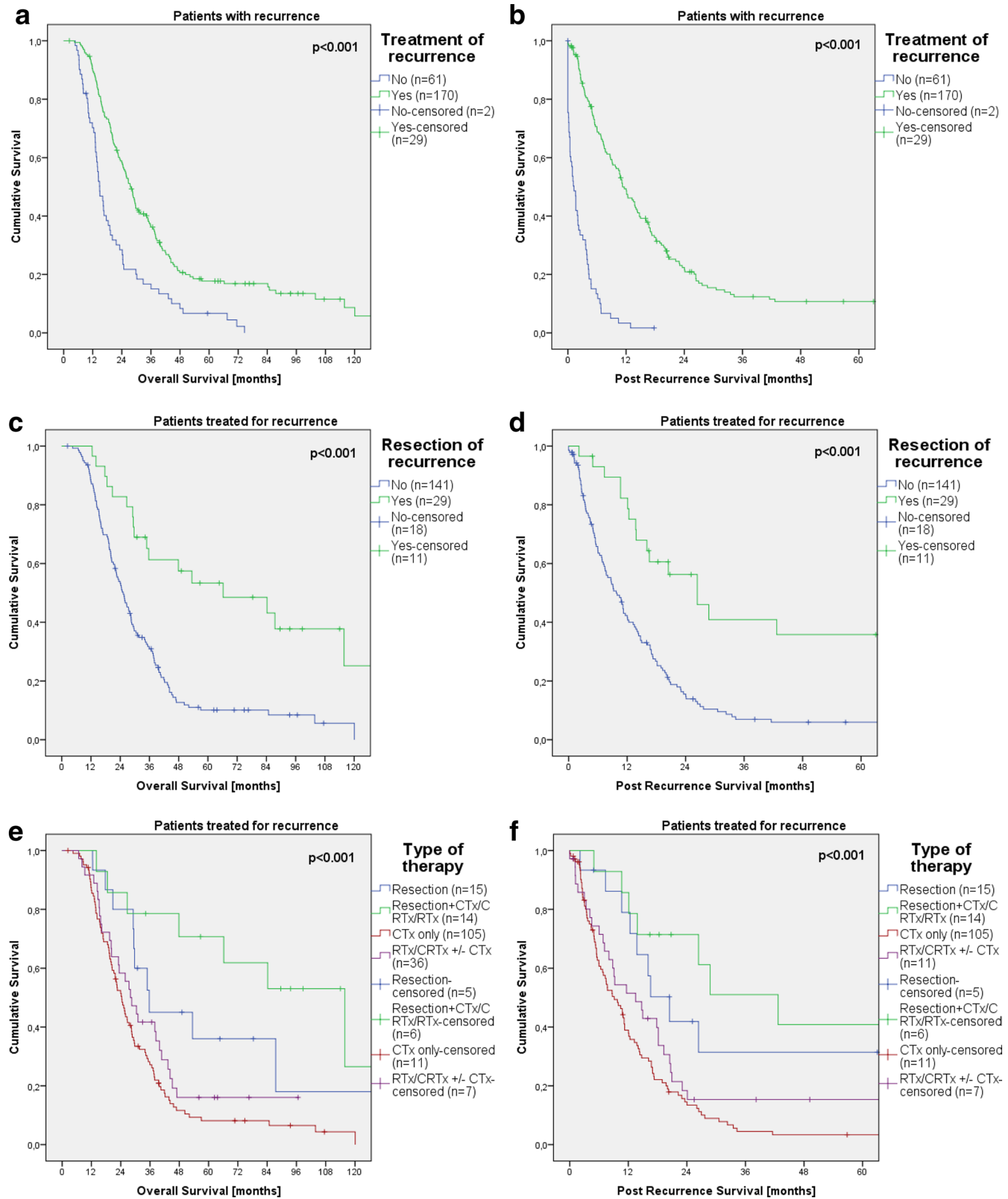

Fig. 2 Influence of treatment of recurrence on a overall survival, b post-recurrence survival; influence of resection of recurrence on $\mathbf{c}$ overall survival, d post-recurrence survival; influence of type of treatment for recurrence on e overall survival, $\mathbf{f}$ post-recurrence survival 
Table 5 Influence of diagnosis, pattern, and treatment of recurrence on overall and postrecurrence survival

\begin{tabular}{|c|c|c|c|c|c|c|c|c|}
\hline & $n$ & Events & $\mathrm{mOS}$ & $95 \% \mathrm{CI}$ & $p$ & $\mathrm{mPRS}$ & $95 \% \mathrm{CI}$ & $p$ \\
\hline Interval till recurrence (months) & & & & & $<0.001$ & & & 0.262 \\
\hline$<12$ & 128 & 118 & 15.2 & $13.7-16.6$ & & 6.9 & $4.8-8.9$ & \\
\hline $12-24$ & 62 & 53 & 27.5 & $23.5-31.6$ & & 6.8 & $1.8-11.8$ & \\
\hline $24-36$ & 24 & 17 & 44.2 & $42.5-45.9$ & & 11.2 & $0.0-22.8$ & \\
\hline$>36$ & 17 & 12 & 74.6 & $53.7-95.5$ & & 9.1 & $3.8-14.4$ & \\
\hline Symptomatic recurrence & & & & & 0.026 & & & $<0.001$ \\
\hline No & 100 & 83 & 27.5 & $23.6-31.5$ & & 13.8 & $10.4-17.2$ & \\
\hline Yes & 87 & 80 & 20.1 & $14.8-25.4$ & & 4.5 & $2.9-6.2$ & \\
\hline Diagnostic means & & & & & 0.010 & & & 0.042 \\
\hline Clinically/sonography & 18 & 17 & 19.4 & $3.9-34.9$ & & 3.7 & $1.8-5.5$ & \\
\hline Endoscopy & 7 & 6 & 12.4 & $11.2-13.7$ & & 2.2 & $1.7-2.7$ & \\
\hline Imaging (CT or MRI) & 153 & 132 & 24.4 & $19.5-29.3$ & & 9.1 & $6.4-11.9$ & \\
\hline Imaging and endoscopy & 37 & 29 & 39.9 & $26.1-53.7$ & & 11.1 & $5.0-17.3$ & \\
\hline Cross-sectional imaging & & & & & 0.011 & & & 0.007 \\
\hline No & 25 & 23 & 22.1 & $5.1-27.9$ & & 3.7 & $2.6-4.7$ & \\
\hline Yes (CT or MRI) & 190 & 161 & 28.5 & $22.8-29.2$ & & 9.2 & $6.8-11.6$ & \\
\hline Histologically confirmed & & & & & 0.007 & & & 0.091 \\
\hline No & 115 & 102 & 22.1 & $17.9-26.3$ & & 6.8 & $4.0-9.6$ & \\
\hline Yes & 100 & 82 & 28.5 & $24.8-32.2$ & & 10.7 & $7.9-13.4$ & \\
\hline Locoregional recurrence & & & & & 0.127 & & & 0.626 \\
\hline No & 151 & 130 & 23.3 & $19.9-26.8$ & & 7.3 & $4.2-10.3$ & \\
\hline Yes & 65 & 55 & 31.1 & $22.2-39.9$ & & 9.1 & $5.1-13.1$ & \\
\hline Distant recurrence & & & & & 0.191 & & & 0.190 \\
\hline No & 24 & 19 & 35.9 & $21.9-49.9$ & & 14.8 & $9.5-20.0$ & \\
\hline Yes & 192 & 166 & 24.2 & $20.9-27.6$ & & 7.5 & $5.4-9.6$ & \\
\hline Peritoneal recurrence & & & & & 0.004 & & & $<0.001$ \\
\hline No & 145 & 121 & 27.5 & $23.3-31.7$ & & 11.1 & $7.8-14.5$ & \\
\hline Yes & 71 & 64 & 20.4 & $13.5-27.3$ & & 6.0 & $4.2-7.7$ & \\
\hline Multiple sites & & & & & 0.248 & & & 0.013 \\
\hline No & 115 & 97 & 25.8 & $21.7-30.0$ & & 12.1 & $9.0-15.2$ & \\
\hline Yes & 101 & 88 & 22.6 & $17.6-27.6$ & & 6.0 & $3.9-8.2$ & \\
\hline Treatment of recurrence & & & & & $<0.001$ & & & $<0.001$ \\
\hline No & 61 & 59 & 14.9 & $12.3-17.4$ & & 1.2 & $0.5-2.0$ & \\
\hline Yes & 170 & 141 & 27.5 & $24.7-30.4$ & & 11.2 & $9.0-13.4$ & \\
\hline Intention of treatment & & & & & 0.001 & & & $<0.001$ \\
\hline Palliative & 145 & 126 & 25.6 & $22.2-28.9$ & & 10.0 & $8.0-12.0$ & \\
\hline Curative & 25 & 15 & 47.9 & $22.3-73.5$ & & 26.4 & $18.8-34.0$ & \\
\hline Resection of recurrence & & & & & $<0.001$ & & & $<0.001$ \\
\hline No (CTx/CRTx/RTx only) & 141 & 123 & 25.2 & $21.6-28.9$ & & 9.8 & $7.3-12.3$ & \\
\hline Yes $( \pm \mathrm{CTx} / \mathrm{CRTx} / \mathrm{RTx})$ & 29 & 18 & 66.2 & $17.3-115.2$ & & 26.4 & $15.6-37.2$ & \\
\hline Type of therapy & & & & & $<0.001$ & & & $<0.001$ \\
\hline Resection & 15 & 10 & 35.7 & $25.4-46.1$ & & 20.5 & $13.0-27.9$ & \\
\hline Resection + CTx/CRTx/RTx & 14 & 8 & 115.8 & $60.7-171.0$ & & 42.7 & $19.2-66.2$ & \\
\hline CTx only & 105 & 94 & 24.4 & $21.2-27.6$ & & 9.1 & $6.2-12.1$ & \\
\hline CRTx/RTx $( \pm \mathrm{CTx})$ & 36 & 29 & 28.0 & $21.2-34.8$ & & 13.5 & $6.7-20.3$ & \\
\hline
\end{tabular}

Bold values indicate a significance-level of $p<0.05$

$m O S$ median overall survival, $m P R S$ median post-recurrence survival, $C T$ computed tomography, MRI magnetic resonance imaging, $C T x$ chemotherapy, $C R T x$ chemoradiotherapy, $R T x$ radiotherapy, + and $/=$ or \pm with or without 
imaging is more likely to detect recurrence, and the role of routine endoscopy for postoperative surveillance seems to be limited. In this respect, our findings are in line with recent reviews of the literature $[23,40]$ and studies of other high-volume centers [42, 45, 56, 57]. Moreover, local recurrence is very likely to present with symptoms. A large series at Memorial Sloan-Kettering Cancer Center with 1147 patients could show that $65 \%$ of local recurrences were initially suspected because of symptoms and only $1 \%$ of asymptomatic recurrences were detected by endoscopy [57]. We thus suggest that postoperative surveillance should be based mainly on cross-sectional imaging, whereas routine endoscopy during follow-up should be reserved for patients with high risk of local recurrence, for example, in case of unclear or positive resection margins ( $\mathrm{Rx}$ or R1), though it should be performed in a timely manner in case of local symptoms.

In our patient collective, more than $50 \%$ of patients relapsed within 1 year, $80 \%$ of patients within 2 years, and more than $90 \%$ within 3 years after curative resection. Comparable results have been reported previously in esophagogastric adenocarcinoma [42]. Since only a small percentage of patients will recur more than 3 years postoperatively, and the probability of relapse after more than 5 years is extremely low [3, 24, 50], intensive follow-up should be performed for a minimum of two and maximum of 3 years after surgery, followed by a subsequent de-escalation and discontinuation of surveillance after 5 years.

Our analyses as well as previous studies have shown that the risk for recurrence increases with advanced stage of disease [58-61]. Indeed, for early gastric cancer thousands of follow-up examinations would be necessary to detect relapse as the incidence is very low [1,2]. For these cases, the cost-benefit ratio seems to be unfavorable. On the other hand, in case of locally advanced and nodal-positive esophagogastric adenocarcinoma, intensive follow-up after resection should be more effective, since the probability of relapse is far higher [3-5]. It thus seems reasonable to adapt the intensity of surveillance according to tumor stage.

The lack of curative treatment options for the vast majority of patients with recurrent disease has been frequently used as an argument against regular surveillance after resection of esophagogastric adenocarcinoma. However, our results indicate a strong survival benefit by treatment of recurrent disease. Part of this survival advantage surely is due to the fact that only patients with a good performance status will receive treatment for relapse, while those with poor general status and hence limited prognosis will not. Yet, this certainly does not account for the entire effect of treatment on survival in our collective. Even though the majority of patients received palliative treatment, mostly in the form of chemotherapy, various randomized controlled trials have shown that palliative chemotherapy improves the outcome in esophagogastric cancer
$[27,62,63]$. We could show that patients followed by a specialized center according to a standardized protocol receive treatment after relapse more frequently than those with individual follow-up. Our results indicate that earlier detection of asymptomatic recurrence and hence better performance status might enable a larger proportion of patients to receive treatment with a beneficial effect on prognosis.

The fact that resection of recurrence improved survival is a classical selection bias and therefore not surprising.

Resection of recurrence led to major improvement of prognosis and even long-term survival, especially when combined with further treatment modalities. Since surgical resection was possible only in a small percentage of selected patients with either isolated local recurrence or circumscribed metastatic disease, the detected survival benefit by resection of relapse probably is caused by selection bias to a relevant extent. However, some smaller series have described an improved and even long-term outcome after resection of local recurrence [64-67] and metachronous liver metastases [68-74] of gastric adenocarcinoma as an individual treatment strategy for a small and highly selected subset of patients. Thus, considering the lack of other curative treatment options, surgical treatment should be attempted whenever resection of recurrent lesions seems feasible and performance status is adequate. As in primary esophagogastric adenocarcinoma, surgical resection embedded in multimodal treatment plans seems to offer the best chance of re-cure.

Limitations of the study are the retrospective, non-randomized, and single-center design. Moreover, since the SFU and non-SFU groups were heterogeneous, comparison of prognosis between the two groups has to be interpreted carefully. Even though propensity score matching was performed, remnant heterogeneity between groups cannot be excluded. Besides, despite the considerable sample size of the entire study population, it might still be too small for adequate subgroup analyses.

Despite all limitations, this study strongly supports an intensified standardized follow-up in curatively resected esophagogastric cancer patients. Prospective randomized controlled data on this topic are needed. However, international expert opinion states that attaining high-grade evidence for standardized follow-up by randomized controlled trials is improbable [24]. As long as no prospective data are available, we suggest that all patients should be subjected to an intensified standardized surveillance protocol after curative resection of esophagogastric adenocarcinoma. In summary, we propose postoperative follow-up visits including scheduled cross-sectional imaging as follows: for locally advanced [(y)pT3/4] and/ or nodal positive disease [(y)pN1/2/3]: every 3 months during the first 2 years, every 6 months during the $3 \mathrm{rd}$ and 4th year, and every 12 months during the 5th year; 
for locally limited tumors (pT0/1/2) and nodal negative disease (pN0): every 6 months during the first 3 years and every 12 months during the 4th and 5th year. After complete tumor resection, routine endoscopy is not necessary, but endoscopic examinations should be performed in case of local symptoms.

\section{Conclusion}

Intensive follow-up by a specialized center was associated with improved overall survival in a large propensity score-matched patient collective after complete resection of esophagogastric adenocarcinoma. Cross-sectional imaging seems to be the diagnostic modality of choice for postoperative follow-up. Moreover, the treatment and especially the resection of recurrence were associated with improved survival, treatment being offered significantly more often to patients followed by a specialized center. Since more than $90 \%$ of relapses occur within the first 3 years after surgery, we recommend intensive follow-up by cross-sectional imaging during this time period, whereas routine endoscopy is not required. Follow-up should be stage-adapted, gradually decreased in intensity, and discontinued after 5 years. Early detection of asymptomatic recurrence might allow for a larger proportion of patients to receive treatment and could even facilitate resection of recurrence potentially resulting in repeated cure with long-term survival in a small subgroup of selected patients. National practice guidelines should be modified accordingly.

Acknowledgements Leila Sisic was sponsored by the medical faculty of the University of Heidelberg in the context of a research scholarship. This funding had no involvement in study design; in collection, analysis, or interpretation of data; in writing of the report; and in the decision to submit the article.

\section{Compliance with ethical standards}

Human rights All procedures followed were in accordance with the ethical standards of the responsible committee on human experimentation (institutional and national) and with the Helsinki Declaration of 1964 and later versions.

Informed consent Informed consent or substitute for it was obtained from all patients for being included in the study.

Conflict of interest The authors declare that they do not have any commercial interest in the subject of study.GM Haag reports fees for advisory role from Sanofi, Roche, Taiho, Nordic, Lilly, Pfizer, honoraria from Roche, travel grants from Amgen, Ipsen and Celgene; research funding is provided by Nordic and Taiho Pharmaceuticals. There is no relationship to the submitted work.

\section{References}

1. Sano T, Sasako M, Kinoshita T, Maruyama K. Recurrence of early gastric cancer. Follow-up of 1475 patients and review of the Japanese literature. Cancer. 1993;72(11):3174-8 (Epub 1993/12/01).

2. Song J, Lee HJ, Cho GS, Han SU, Kim MC, Ryu SW, et al. Recurrence following laparoscopy-assisted gastrectomy for gastric cancer: a multicenter retrospective analysis of 1,417 patients. Ann Surg Oncol. 2010;17(7):1777-86. doi:10.1245/s10434-0100932-4 (Epub 2010/02/13).

3. D'Angelica M, Gonen M, Brennan MF, Turnbull AD, Bains M, Karpeh MS. Patterns of initial recurrence in completely resected gastric adenocarcinoma. Ann Surg. 2004;240(5):808-16 (Epub 2004/10/20).

4. Schwarz RE, Zagala-Nevarez K. Recurrence patterns after radical gastrectomy for gastric cancer: prognostic factors and implications for postoperative adjuvant therapy. Ann Surg Oncol. 2002;9(4):394-400 (Epub 2002/05/03).

5. Chiang CY, Huang KH, Fang WL, Wu CW, Chen JH, Lo SS, et al. Factors associated with recurrence within 2 years after curative surgery for gastric adenocarcinoma. World J Surg. 2011;35(11):2472-8. doi:10.1007/s00268-011-1247-8 (Epub 2011/09/01).

6. Chang JS, Lim JS, Noh SH, Hyung WJ, An JY, Lee YC, et al. Patterns of regional recurrence after curative D2 resection for stage III (N3) gastric cancer: implications for postoperative radiotherapy. Radiother Oncol. 2012;104(3):367-73. doi:10.1016/j. radonc.2012.08.017 (Epub 2012/09/18).

7. Honore C, Goere D, Messager M, Souadka A, Dumont F, Piessen $\mathrm{G}$, et al. Risk factors of peritoneal recurrence in eso-gastric signet ring cell adenocarcinoma: results of a multicentre retrospective study. Eur J Surg Oncol. 2013;39(3):235-41. doi:10.1016/j. ejso.2012.12.013 (Epub 2013/01/15).

8. Fields RC, Strong VE, Gonen M, Goodman KA, Rizk NP, Kelsen DP, et al. Recurrence and survival after pathologic complete response to preoperative therapy followed by surgery for gastric or gastrooesophageal adenocarcinoma. Br J Cancer. 2011;104(12):1840-7. doi:10.1038/bjc.2011.175 (Epub 2011/05/26).

9. Ott K, Blank S, Becker K, Langer R, Weichert W, Roth W, et al. Factors predicting prognosis and recurrence in patients with esophago-gastric adenocarcinoma and histopathological response with less than $10 \%$ residual tumor. Langenbeck's Arch Surg. 2013;398(2):239-49. doi:10.1007/s00423-012-1039-0 (Epub 2012/12/28).

10. Schmidt T, Sicic L, Blank S, Becker K, Weichert W, Bruckner $\mathrm{T}$, et al. Prognostic value of histopathological regression in 850 neoadjuvantly treated oesophagogastric adenocarcinomas. Br J Cancer. 2014;110(7):1712-20. doi:10.1038/bjc.2014.94 (Epub 2014/02/27).

11. Springfeld C, Wiecha C, Kunzmann R, Heger U, Weichert W, Langer R, et al. Influence of different neoadjuvant chemotherapy regimens on response, prognosis, and complication rate in patients with esophagogastric adenocarcinoma. Ann Surg Oncol. 2015;22(Suppl 3):S905-14. doi:10.1245/s10434-0154617-x (Epub 2015/05/24).

12. Ajani JA, Barthel JS, Bekaii-Saab T, Bentrem DJ, D’Amico TA, Das P, et al. Gastric cancer. J Natl Compr Cancer Netw. 2010;8(4):378-409 (Epub 2010/04/23).

13. Japanese Gastric Cancer A. Japanese gastric cancer treatment guidelines 2014 (ver. 4). Gastric Cancer. 2017;20(1):1-19. doi:10.1007/s10120-016-0622-4 (Epub 2016/06/28).

14. Allum WH, Blazeby JM, Griffin SM, Cunningham D, Jankowski JA, Wong R, et al. Guidelines for the management 
of oesophageal and gastric cancer. Gut. 2011;60(11):1449-72. doi:10.1136/gut.2010.228254 (Epub 2011/06/28).

15. Waddell T, Verheij M, Allum W, Cunningham D, Cervantes A, Arnold D. Gastric cancer: ESMO-ESSO-ESTRO clinical practice guidelines for diagnosis, treatment and follow-up. Ann Oncol. 2013;24(Suppl 6):vi57-63. doi:10.1093/annonc/ mdt344 (Epub 2013/10/23).

16. Moehler M, Al-Batran SE, Andus T, Anthuber M, Arends $\mathrm{J}$, Arnold D, et al. German S3-guideline "Diagnosis and treatment of esophagogastric cancer". Z Gastroenterol. 2011;49(4):461-531. doi:10.1055/s-0031-1273201 (Epub 2011/04/09).

17. Marrelli D, De Stefano A, de Manzoni G, Morgagni P, Di Leo A, Roviello F. Prediction of recurrence after radical surgery for gastric cancer: a scoring system obtained from a prospective multicenter study. Ann Surg. 2005;241(2):247-55 (Epub 2005/01/15).

18. Peeters M, Lerut T, Vlayen J, Mambourg F, Ectors N, Deprez $\mathrm{P}$, et al. Wetenschappelijke ondersteuning van het College voor Oncologie: een nationale praktijkrichtlijn voor de aanpak van slokdarm- en maagkanker. 2008.

19. Park MJ, Lee WJ, Lim HK, Park KW, Choi JY, Kim BT. Detecting recurrence of gastric cancer: the value of FDG PET/CT. Abdom Imaging. 2009;34(4):441-7. doi:10.1007/s00261-0089424-4 (Epub 2008/06/11).

20. Sun L, Su XH, Guan YS, Pan WM, Luo ZM, Wei JH, et al. Clinical role of $18 \mathrm{~F}$-fluorodeoxyglucose positron emission tomography/computed tomography in post-operative follow up of gastric cancer: initial results. World J Gastroenterol. 2008;14(29):462732 (Epub 2008/08/14).

21. Wu LM, Hu JN, Hua J, Gu HY, Zhu J, Xu JR. 18 F-fluorodeoxyglucose positron emission tomography to evaluate recurrent gastric cancer: a systematic review and meta-analysis. J Gastroenterol Hepatol. 2012;27(3):472-80. doi:10.1111/j.14401746.2011.06919.x (Epub 2011/09/16).

22. Messager M, de Steur W, Boelens PG, Jensen LS, Mariette C, Reynolds JV, et al. Description and analysis of clinical pathways for oesophago-gastric adenocarcinoma, in 10 European countries (the EURECCA upper gastro intestinal group-European Registration of Cancer Care). Eur J Surg Oncol. 2016;42(9):1432-47. doi:10.1016/j.ejso.2016.01.001 (Epub 2016/02/24).

23. Nilsson M. Postgastrectomy follow-up in the west: evidence base, guidelines, and daily practice. Gastric Cancer. 2017;20(Suppl 1):135-40. doi:10.1007/s10120-016-0654-9 (Epub 2016/10/09).

24. Baiocchi GL, D'Ugo D, Coit D, Hardwick R, Kassab P, Nashimoto A, et al. Follow-up after gastrectomy for cancer: the Charter Scaligero Consensus Conference. Gastric Cancer. 2016;19(1):15-20. doi:10.1007/s10120-015-0513-0 (Epub 2015/07/05).

25. Lorenzen S, Hentrich M, Haberl C, Heinemann V, Schuster T, Seroneit T, et al. Split-dose docetaxel, cisplatin and leucovorin/ fluorouracil as first-line therapy in advanced gastric cancer and adenocarcinoma of the gastroesophageal junction: results of a phase II trial. Ann Oncol. 2007;18(10):1673-9.

26. Al-Batran SE, Hartmann JT, Hofheinz R, Homann N, Rethwisch $\mathrm{V}$, Probst S, et al. Biweekly fluorouracil, leucovorin, oxaliplatin, and docetaxel (FLOT) for patients with metastatic adenocarcinoma of the stomach or esophagogastric junction: a phase II trial of the Arbeitsgemeinschaft Internistische Onkologie. Ann Oncol. 2008;19(11):1882-7.

27. Van Cutsem E, Moiseyenko VM, Tjulandin S, Majlis A, Constenla M, Boni C, et al. Phase III study of docetaxel and cisplatin plus fluorouracil compared with cisplatin and fluorouracil as first-line therapy for advanced gastric cancer: a report of the V325 Study Group. J Clin Oncol. 2006;24(31):4991-7. doi:10.1200/JCO.2006.06.8429 (Epub 2006/11/01).
28. Bang YJ, Van Cutsem E, Feyereislova A, Chung HC, Shen L, Sawaki A, et al. Trastuzumab in combination with chemotherapy versus chemotherapy alone for treatment of HER2-positive advanced gastric or gastro-oesophageal junction cancer (ToGA): a phase 3, open-label, randomised controlled trial. Lancet. 2010;376(9742):687-97. doi:10.1016/S0140-6736(10)61121-X (Epub 2010/08/24)

29. Wilke H, Muro K, Van Cutsem E, Oh SC, Bodoky G, Shimada Y, et al. Ramucirumab plus paclitaxel versus placebo plus paclitaxel in patients with previously treated advanced gastric or gastro-oesophageal junction adenocarcinoma (RAINBOW): a double-blind, randomised phase 3 trial. Lancet Oncol. 2014;15(11):1224-35. doi:10.1016/S1470-2045(14)70420-6 (Epub 2014/09/23)

30. Sakamoto Y, Sano T, Shimada K, Esaki M, Saka M, Fukagawa T, et al. Favorable indications for hepatectomy in patients with liver metastasis from gastric cancer. J Surg Oncol. 2007;95(7):534-9. doi:10.1002/jso.20739 (Epub 2007/01/16).

31. Lim S, Muhs BE, Marcus SG, Newman E, Berman RS, Hiotis SP. Results following resection for stage IV gastric cancer; are better outcomes observed in selected patient subgroups? J Surg Oncol. 2007;95(2):118-22. doi:10.1002/jso.20328 (Epub 2007/01/31).

32. Li C, Yan M, Zhu ZG. Nonpalliative surgical resection for gastric cancer patients with distant metastasis. J Investig Surg. 2012;25(2):100-6. doi:10.3109/08941939.2011.607225 (Epub 2012/03/24)

33. Kodera Y, Fujitani K, Fukushima N, Ito S, Muro K, Ohashi N, et al. Surgical resection of hepatic metastasis from gastric cancer: a review and new recommendation in the Japanese gastric cancer treatment guidelines. Gastric Cancer. 2013;. doi:10.1007/ s10120-013-0299-x (Epub 2013/09/12).

34. Cheon SH, Rha SY, Jeung HC, Im CK, Kim SH, Kim HR, et al. Survival benefit of combined curative resection of the stomach (D2 resection) and liver in gastric cancer patients with liver metastases. Ann Oncol. 2008;19(6):1146-53. doi:10.1093/ annonc/mdn026 (Epub 2008/02/29).

35. Schmidt T, Alldinger I, Blank S, Klose J, Springfeld C, Dreikhausen L, et al. Surgery in oesophago-gastric cancer with metastatic disease: treatment, prognosis and preoperative patient selection. Eur J Surg Oncol. 2015;41(10):1340-7. doi:10.1016/j. ejso.2015.05.005 (Epub 2015/07/28).

36. Yonemura Y, Kawamura T, Bandou E, Takahashi S, Sawa T, Matsuki N. Treatment of peritoneal dissemination from gastric cancer by peritonectomy and chemohyperthermic peritoneal perfusion. Br J Surg. 2005;92(3):370-5. doi:10.1002/bjs.4695 (Epub 2005/03/02).

37. Yonemura Y, Bandou E, Sawa T, Yoshimitsu Y, Endou Y, Sasaki T, et al. Neoadjuvant treatment of gastric cancer with peritoneal dissemination. Eur J Surg Oncol. 2006;32(6):661-5. doi:10.1016/j.ejso.2006.03.007 (Epub 2006/04/20).

38. Hall JJ, Loggie BW, Shen P, Beamer S, Douglas Case L, McQuellon R, et al. Cytoreductive surgery with intraperitoneal hyperthermic chemotherapy for advanced gastric cancer. J Gastrointest Surg. 2004;8(4):454-63. doi:10.1016/j.gassur.2003.12.014 (Epub 2004/05/04).

39. Cheong JH, Shen JY, Song CS, Hyung WJ, Shen JG, Choi SH, et al. Early postoperative intraperitoneal chemotherapy following cytoreductive surgery in patients with very advanced gastric cancer. Ann Surg Oncol. 2007;14(1):61-8. doi:10.1245/s10434-0069205-7 (Epub 2006/10/24).

40. Cardoso R, Coburn NG, Seevaratnam R, Mahar A, Helyer L, Law C, et al. A systematic review of patient surveillance after curative gastrectomy for gastric cancer: a brief review. Gastric Cancer. 2012;15(Suppl 1):S164-7. doi:10.1007/s10120-0120142-9 (Epub 2012/03/03). 
41. Moyes LH, Anderson JE, Forshaw MJ. Proposed follow up programme after curative resection for lower third oesophageal cancer. World J Surg Oncol. 2010;8:75. doi:10.1186/1477-7819-875 (Epub 2010/09/08).

42. Moorcraft SY, Fontana E, Cunningham D, Peckitt C, Waddell T, Smyth EC, et al. Characterising timing and pattern of relapse following surgery for localised oesophagogastric adenocarcinoma: a retrospective study. BMC Cancer. 2016;16:112. doi:10.1186/ s12885-016-2145-0 (Epub 2016/02/18).

43. Park CH, Park JC, Chung H, Shin SK, Lee SK, Cheong JH, et al. Impact of the surveillance interval on the survival of patients who undergo curative surgery for gastric cancer. Ann Surg Oncol. 2016;23(2):539-45. doi:10.1245/s10434-015-4866-8 (Epub 2015/10/02).

44. Peixoto RD, Lim HJ, Kim H, Abdullah A, Cheung WY. Patterns of surveillance following curative intent therapy for gastroesophageal cancer. J Gastrointest Cancer. 2014;45(3):325-33. doi:10.1007/s12029-014-9601-3 (Epub 2014/04/24).

45. Tan IT, So BY. Value of intensive follow-up of patients after curative surgery for gastric carcinoma. J Surg Oncol. 2007;96(6):503-6. doi:10.1002/jso.20823 (Epub 2007/08/08).

46. Hur H, Song KY, Park CH, Jeon HM. Follow-up strategy after curative resection of gastric cancer: a nationwide survey in Korea. Ann Surg Oncol. 2010;17(1):54-64. doi:10.1245/s10434009-0676-1 (Epub 2009/09/25).

47. Whiting J, Sano T, Saka M, Fukagawa T, Katai H, Sasako M. Follow-up of gastric cancer: a review. Gastric Cancer. 2006;9(2):74-81. doi:10.1007/s10120-006-0360-0 (Epub 2006/06/13).

48. Li JH, Zhang SW, Liu J, Shao MZ, Chen L. Review of clinical investigation on recurrence of gastric cancer following curative resection. Chin Med J. 2012;125(8):1479-95 (Epub 2012/05/23).

49. Bali C, Roukos DH, Ziogas DE. Follow-up for gastric cancer: how extensive and intensive should it be? Ann Surg Oncol. 2010;17(3):941. doi:10.1245/s10434-009-0821-x (Epub 2009/12/05; author reply 2).

50. D’Ugo D, Biondi A, Tufo A, Persiani R. Follow-up: the evidence. Diges Surg. 2013;30(2):159-68. doi:10.1159/000350878 (Epub 2013/07/23).

51. Kodera Y, Ito S, Yamamura Y, Mochizuki Y, Fujiwara M, Hibi $\mathrm{K}$, et al. Follow-up surveillance for recurrence after curative gastric cancer surgery lacks survival benefit. Ann Surg Oncol. 2003;10(8):898-902 (Epub 2003/10/07).

52. D’Agostino RB Jr. Propensity score methods for bias reduction in the comparison of a treatment to a non-randomized control group. Stat Med. 1998;17(19):2265-81 (Epub 1998/11/05).

53. Bilici A, Salman T, Oven Ustaalioglu BB, Unek T, Seker M, Aliustaoglu M, et al. The prognostic value of detecting symptomatic or asymptomatic recurrence in patients with gastric cancer after a curative gastrectomy. J Surg Res. 2013;180(1):e1-9. doi:10.1016/j.jss.2012.03.035 (Epub 2012/04/24).

54. Kim JH, Jang YJ, Park SS, Park SH, Mok YJ. Benefit of postoperative surveillance for recurrence after curative resection for gastric cancer. J Gastrointest Surg. 2010;14(6):969-76. doi:10.1007/s11605-010-1200-4 (Epub 2010/04/23).

55. Spolverato G, Ejaz A, Kim Y, Squires MH, Poultsides GA, Fields RC, et al. Rates and patterns of recurrence after curative intent resection for gastric cancer: a US multi-institutional analysis. J Am Coll Surg. 2014;219(4):664-75. doi:10.1016/j.jamcollsurg.2014.03.062 (Epub 2014/08/27).

56. Lee SY, Lee JH, Hwang NC, Kim YH, Rhee PL, Kim JJ, et al. The role of follow-up endoscopy after total gastrectomy for gastric cancer. Eur J Surg Oncol. 2005;31(3):265-9. doi:10.1016/j. ejso.2004.11.018 (Epub 2005/03/23).
57. Lou F, Sima CS, Adusumilli PS, Bains MS, Sarkaria IS, Rusch VW, et al. Esophageal cancer recurrence patterns and implications for surveillance. J Thorac Oncol. 2013;8(12):1558-62. doi:10.1097/01.JTO.0000437420.38972.fb (Epub 2014/01/07).

58. Bilici A, Selcukbiricik F. Prognostic significance of the recurrence pattern and risk factors for recurrence in patients with proximal gastric cancer who underwent curative gastrectomy. Tumour Biol. 2015;36(8):6191-9. doi:10.1007/s13277-0153304-7 (Epub 2015/03/13).

59. Li F, Zhang R, Liang H, Liu H, Quan J. The pattern and risk factors of recurrence of proximal gastric cancer after curative resection. J Surg Oncol. 2013;107(2):130-5. doi:10.1002/jso.23252 (Epub 2012/09/06).

60. Nakagawa M, Kojima K, Inokuchi M, Kato K, Sugita H, Kawano $\mathrm{T}$, et al. Patterns, timing and risk factors of recurrence of gastric cancer after laparoscopic gastrectomy: reliable results following long-term follow-up. Eur J Surg Oncol. 2014;40(10):1376-82. doi:10.1016/j.ejso.2014.04.015 (Epub 2014/06/12).

61. Nakanishi Y, Ohara M, Domen H, Shichinohe T, Hirano S, Ishizaka M. Differences in risk factors between patterns of recurrence in patients after curative resection for advanced gastric carcinoma. World J Surg Oncol. 2013;11:98. doi:10.1186/14777819-11-98 (Epub 2013/05/21).

62. Al-Batran SE, Hartmann JT, Probst S, Schmalenberg H, Hollerbach S, Hofheinz R, et al. Phase III trial in metastatic gastroesophageal adenocarcinoma with fluorouracil, leucovorin plus either oxaliplatin or cisplatin: a study of the Arbeitsgemeinschaft Internistische Onkologie. J Clin Oncol. 2008;26(9):1435-42.

63. Wagner AD, Unverzagt S, Grothe W, Kleber G, Grothey A, Haerting J, et al. Chemotherapy for advanced gastric cancer. Cochrane Database Syst Rev (Online). 2010;3:CD004064. doi:10.1002/14651858.CD004064.pub3 (Epub 2010/03/20).

64. Badgwell B, Cormier JN, Xing Y, Yao J, Bose D, Krishnan S, et al. Attempted salvage resection for recurrent gastric or gastroesophageal cancer. Ann Surg Oncol. 2009;16(1):42-50. doi:10.1245/s10434-008-0210-x (Epub 2008/11/06).

65. de Liano AD, Yarnoz C, Aguilar R, Artieda C, Ortiz H. Surgical treatment of recurrent gastric cancer. Gastric Cancer. 2008;11(1):10-4. doi:10.1007/s10120-007-0444-5 (Epub 2008/04/01).

66. Song KY, Park SM, Kim SN, Park $\mathrm{CH}$. The role of surgery in the treatment of recurrent gastric cancer. Am J Surg. 2008;196(1):19-22. doi:10.1016/j.amjsurg.2007.05.056 (Epub 2008/04/18).

67. Ozer I, Bostanci EB, Ozogul Y, Ulas M, Ercan M, Kece C, et al. Laparotomy with a curative intent in patients with suspected locally recurrent gastric cancer. Tumori. 2009;95(4):438-41 (Epub 2009/10/28).

68. Tiberio GA, Coniglio A, Marchet A, Marrelli D, Giacopuzzi $\mathrm{S}$, Baiocchi L, et al. Metachronous hepatic metastases from gastric carcinoma: a multicentric survey. Eur J Surg Oncol. 2009;35(5):486-91. doi:10.1016/j.ejso.2008.12.017 (Epub 2009/01/28).

69. Koga R, Yamamoto J, Ohyama S, Saiura A, Seki M, Seto Y, et al. Liver resection for metastatic gastric cancer: experience with 42 patients including eight long-term survivors. Jpn J Clin Oncol. 2007;37(11):836-42. doi:10.1093/jjco/hym113 (Epub 2007/10/12).

70. Sakamoto Y, Ohyama S, Yamamoto J, Yamada K, Seki M, Ohta $\mathrm{K}$, et al. Surgical resection of liver metastases of gastric cancer: an analysis of a 17-year experience with 22 patients. Surgery. 2003;133(5):507-11. doi:10.1067/msy.2003.147 (Epub 2003/05/30).

71. Schildberg CW, Croner R, Merkel S, Schellerer V, Muller V, Yedibela $S$, et al. Outcome of operative therapy of hepatic metastatic stomach carcinoma: a retrospective analysis. World J Surg. 
2012;36(4):872-8. doi:10.1007/s00268-012-1492-5 (Epub 2012/02/23)

72. Roh HR, Suh KS, Lee HJ, Yang HK, Choe KJ, Lee KU. Outcome of hepatic resection for metastatic gastric cancer. Am Surg. 2005;71(2):95-9 (Epub 2005/07/19).

73. Choi SB, Song J, Kang CM, Hyung WJ, Kim KS, Choi JS, et al. Surgical outcome of metachronous hepatic metastases secondary to gastric cancer. Hepato-gastroenterology. 2010;57(97):29-34 (Epub 2010/04/29)

74. Zacherl J, Zacherl M, Scheuba C, Steininger R, Wenzl E, Muhlbacher F, et al. Analysis of hepatic resection of metastasis originating from gastric adenocarcinoma. J Gastrointest Surg. 2002;6(5):682-9 (Epub 2002/10/26). 